\title{
Effects of Riboflavin Interactions with 5-Methyltetrahydrofolate and Tetrahydrofolate on Changes in Homocysteine and Folate Derivative Levels, with and without Methionine Addition
}

\author{
Risa Araki, Kiho Imada, Makiko Hikichi, Yukiko Arai, Hihumi Kashima, Chizuko Maruyama* \\ Department of Food and Nutrition, Japan Women's University, Tokyo, Japan. \\ Email: *maruyama@fc.jwu.ac.jp \\ Received June $13^{\text {th }}, 2013$; revised July $13^{\text {th }}, 2013$; accepted July $20^{\text {th }}, 2013$ \\ Copyright (C 2013 Risa Araki et al. This is an open access article distributed under the Creative Commons Attribution License, which \\ permits unrestricted use, distribution, and reproduction in any medium, provided the original work is properly cited.
}

\begin{abstract}
Riboflavin has been suggested to act with folate to lower homocysteine (Hcy). However, these interactions may differ among the several known forms of folate. Therefore, we examined the effects of riboflavin interactions with 5-methyltetrahydrofolate (5-MTHF) and tetrahydrofolate (THF) on changes in Hcy and folate derivative levels, under conditions with and without methionine addition. Rat hepatocytes were cultured for 48 hours in medium with or without $2.64 \mu \mathrm{M}$ riboflavin addition, under the following conditions: 1) without the addition of either methionine or folate; 2) with addition of $2 \mu \mathrm{M}$ folate derivatives [(A): 5-MTHF, (B): THF]; 3) with addition of both $5 \mathrm{mM}$ methionine and 2 $\mu \mathrm{M}$ folate derivatives [(A): 5-MTHF, (B): THF]. The supernatants were collected at 0, 24, and 48 hours for Hcy and folate derivative measurements. The Hcy lowering effect of 5-MTHF, as well as inhibition of 5-MTHF reduction and THF elevation, appeared to be enhanced by riboflavin addition. THF addition did not lower the Hcy level, regardless of the presence of riboflavin and/or methionine, while THF and 5,10-methenyl THF levels were maintained. Further examination is needed to elucidate the interactive effects of riboflavin and folate derivatives on Hcy and folate metabolism.
\end{abstract}

Keywords: Folate Derivatives; Riboflavin; Interaction; Homocysteine

\section{Introduction}

Folate plays an important role in the remethylation of homocysteine (Hcy), and the synthesis of thymidylate and purine which are precursors for DNA and RNA synthesis [1]. Folate derivatives are formed by different onecarbon subgroups. As shown in Figure 1, dietary folate is generally reduced to tetrahydrofolate (THF) and then metabolized to 5-methyltetrahydrofolate (5-MTHF) via 5, 10-methylenetetrahydrofolate reductase (MTHFR) which is a flavoprotein enzyme requiring a flavin adenine dinucleotide (FAD), a derivative of riboflavin, as its coenzyme. 5-MTHF is converted back into THF in parallel with the conversion of Hcy to methionine. A former study examining the effect of riboflavin status on the interaction between the MTHFR TT genotype and Hcy levels in healthy subjects suggested that those with the

*Corresponding author.
TT genotype are particularly sensitive to riboflavin status [2]. The Hcy lowering effect of riboflavin [3] and an inverse association between plasma Hcy and plasma riboflavin levels [4] were reported in subjects with the MTHFR TT genotype. Thus, riboflavin would be expected to enhance the Hcy-lowering effect of folate.

However, in our previous short-term supplementation study, serum 5-MTHF levels were increased by folic acid supplementation, but not by folic acid plus riboflavin supplementation, in healthy young Japanese men. Furthermore, no significant Hcy reduction was observed during the intervention in the group given folic acid combined with riboflavin as compared to the group given folic acid alone [5]. Similar results were reported in healthy men and women approximately 40 years of age [6]. Powers et al. also found that in healthy subjects and colorectal polyp patients who had good overall folate status, riboflavin supplementation did not raise plasma 


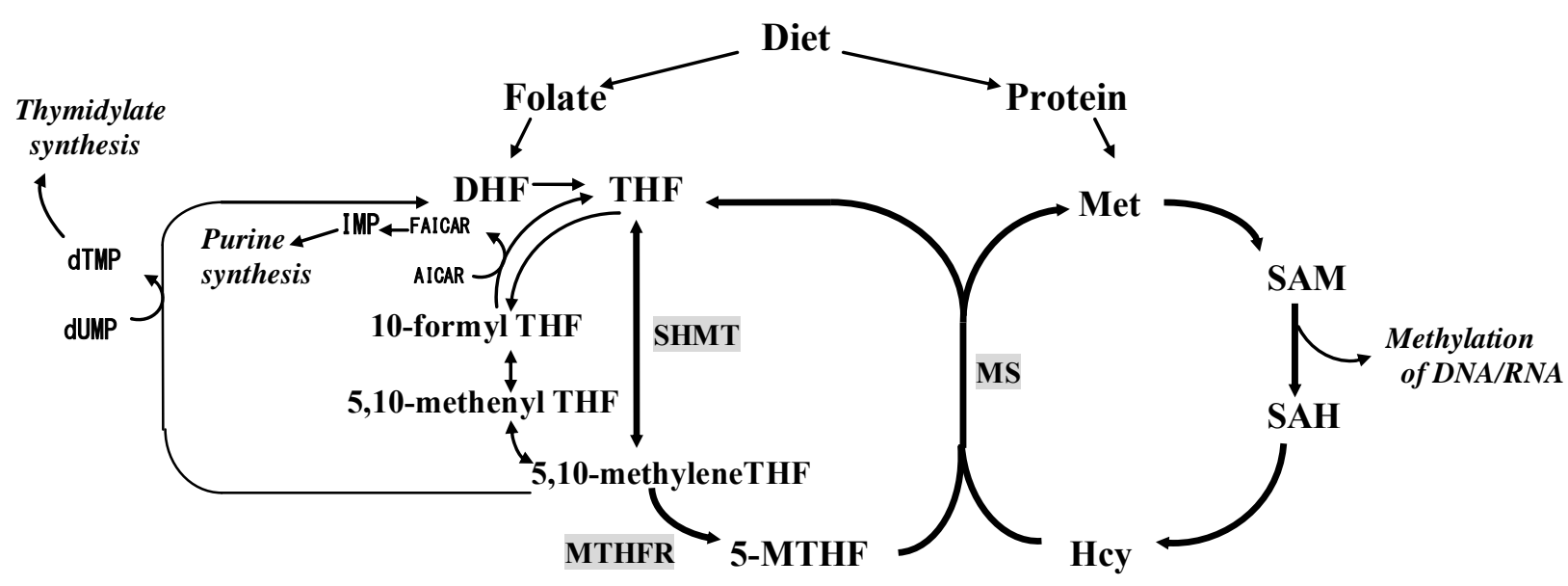

Figure 1. Folate derivatives metabolism. DHF = dihydrofolate; THF = tetrahydrofolate; 5-MTHF = 5-methyltetrahydrofolate; 5,10-metyleneTHF = 5,10-methylenetetrahydrofolate; 5,10-methenyl THF = 5,10-methenyltetrahydrofolate; 10-formyl THF $=$ 10-formyltetrahydrofolate; Hcy = homocysteine; Met = methionine; SAM = s-adenosyl methionine; SAH = s-adenosyl homocysteine; dUMP = deoxyuridine monophosphate; dTMP = deoxythymidine monophosphate; AICAR = 5-amino-4-imidazole carboxamide ribonucleotide; FAICAR = 5-formamidoimidazole-4-carboxamide ribonucleotide; IMP = inosine monophosphate; SHMT = serine hydroxymethyltransferase; MTHFR = methylenetetrahydrofolate reductase; MS = methionine synthase.

5-MTHF and lower Hcy in the healthy subject group, while the plasma 5-MTHF level was increased by riboflavin supplementation in colorectal polyp patients with the CT or the TT genotype [7]. Though consensus is lacking as to whether riboflavin enhances the Hcy lowering effect of folic acid, the effect of combining folic acid and riboflavin supplementation on folate and Hcy metabolism might differ according to by MTHFR mutation status and/or the levels of other factors impacting Hcy, folate, and riboflavin.

It is difficult to demonstrate the effects of riboflavin on folate and Hcy metabolism in a human study because numerous interrelated factors influence these metabolic processes. Thus, a simple cellular system is needed to clarify the effects of riboflavin. Earlier studies demonstrating the combined effects of folic acid and riboflavin were performed using lymphocytes [8,9]. The liver is reportedly the major site of methionine catabolism [10], and hepatocytes can make a major contribution to the plasma Hcy level [11]. Though folate and riboflavin presumably interact in hepatocytes, no prior study has yet used hepatocytes to examine this interaction.

Among folate derivatives, 5-MTHF and THF are the two dominant circulating forms in mammals [1]. Thus, the combined effects of folate derivatives and riboflavin on Hcy and folate metabolism should be examined not only for 5-MTHF but also for THF. However, the interactive effects of THF and riboflavin on Hcy and folate metabolism have yet to be elucidated. On the other hand, THF derived from food is considered to be reversibly metabolized to 5,10 -methylene THF by not only serine hydroxymethyltransferase but also via 10-formyl THF and 5,10-methenyl THF. Then 5,10-methylene THF is converted to 5-MTHF by the flavoprotein enzyme MTHFR. Furthermore, in the state of reduced MTHFR activity, 5,10-methylene THF is suggested to be converted back to THF in the pathway including 5,10methenyl THF and 10-formyl THF [12].

Thus, changes in THF and 5,10-methylene THF levels are useful for estimating the effects of riboflavin on 5MTHF production. However, 5,10-methylene THF cannot be measured because of its instability [13], while 5,10-methenyl THF is measurable.

Therefore, we examined the effects of riboflavin interactions with 5-MTHF and THF on changes in Hcy, 5MTHF, THF and 5,10-methenyl THF levels in rat hepatocytes, under conditions with and without methionine addition.

\section{Materials and Methods}

\subsection{Materials}

Medium 199 was obtained from Invitrogen (Netherlands). Riboflavin was obtained from Sigma Aldrich Japan (Japan). 6S-5-metyltetrahydrofolate, 6S-tetrahydrofolate, and 6S-5,10-methenyltetrahydrofolate were obtained from Shircks Laboratories (Switzerland).

\subsection{Methods}

\subsubsection{Cell Culture Conditions}

In this experiment, hepatocytes (Culture Kit P-4 of Primary Cell, Japan) were used. This kit consisted of hepatocytes isolated from Sprague-Dawley rat livers, and 
seeded at $2 \times 10^{5}$ cells/well in $0.5 \mathrm{ml}$ of Dulbecco's modified Eagle medium supplemented with $10 \%$ fetal calf serum. The cultures were kept in a humidified incubator with $5 \% \mathrm{CO}_{2}$ and maintained at $37^{\circ} \mathrm{C}$. After 2 hours of incubation to allow adherence, the medium was gently removed. The cells were then pre-incubated for 24 hours in the basal medium; Medium 199 (containing $26 \mathrm{nM}$ riboflavin, $20 \mathrm{nM}$ folic acid and $0.1 \mathrm{mM}$ methionine) supplemented with $10 \%$ fetal calf serum and $1.5 \mu \mathrm{M}$ cyanocobalamin according to a slight modification of the culture conditions described by Christensen et al. [14]. After pre-incubation, cell cultures were performed for 48 hours in the incubation medium. The basal medium mentioned above was used with or without $2.64 \mu \mathrm{M}$ riboflavin addition, under the following conditions: 1 ) without the addition of either methionine or folate; 2 ) with the addition of $2 \mu \mathrm{M}$ folate derivatives [(A): 5-MTHF, (B): THF]; 3) with the addition of $5 \mathrm{mM}$ methionine and 2 $\mu \mathrm{M}$ folate derivatives [(A): 5-MTHF, (B): THF]. We determined the amounts of folate and riboflavin to be added as mentioned above, to assure that these levels would be the same in Eagle's minimal essential medium (EMEM) which was used in the former study assessing the effects of methionine on changes in Hcy levels in hepatocytes [14]. The results of the preliminary experiments, conducted to determine the optimal amount of methionine under the conditions of adding $2 \mu \mathrm{M}$ folate or $2.64 \mu \mathrm{M}$ riboflavin, revealed $5 \mathrm{mM}$ methionine to be useful for examining Hcy level changes.

The supernatants were collected at 0,24 and 48 hours, centrifuged to remove cells, and the medium was frozen at $-80^{\circ} \mathrm{C}$ until determination of folate derivatives and Hcy.

\subsubsection{Analysis of Folate Derivatives in Supernatants}

The 5-MTHF, THF, and 5,10-methenyl THF levels in supernatants were determined using high-performance liquid chromatography (HPLC) with the JASCO HPLC system (Jasco, Japan). Each sample was placed in a glove bag filled with nitrogen gas shielded from light, and applied to a COSMOSIL (R) 5PE-MS Packed Column (4.6 mm I.D. $\times 150 \mathrm{~mm}$ ) (Nacalai Tesque, Japan). The mobile phase was a solution of $50 \mathrm{mM}$ potassium dihydrogen phosphate (pH3.5) containing $0.1 \mathrm{mM}$ ethylene diamine tetraacetic acid disodium salt, dehydrated in 5\% methanol, with the flow rate set at $1.0 \mathrm{~mL} / \mathrm{min}$. Detection was performed using a UV 970 (Jasco, Japan) at $280 \mathrm{~nm}$.

\subsubsection{Analysis of Hcy in Supernatants}

The Hcy levels in supernatants were determined by reverse-phase HPLC and fluorescence detection with a Shimadzu HPLC system (Shimadzu, Japan). HPLC was performed on a CAPCEL PAK C18 UG120 (4.6 mm I.D. $\times 100 \mathrm{~mm}$ ) (Shiseido, Japan) using $0.1 \mathrm{M}$ potassium di- hydrogen phosphate with $5 \%$ methanol, adjusted to $\mathrm{pH}$ 2.7 with phosphoric acid as a mobile phase with a flow rate of $1.0 \mathrm{~mL} / \mathrm{min}$. Detection was performed at an excitation wavelength of $385 \mathrm{~nm}$ and an emission wavelength of $515 \mathrm{~nm}$. As a reducing reagent, tris-(2-carboxy-ethyl)phosphine was used, with 4-fluoro-7-sulfobenzo-furazan, ammonium salt serving as the derivatization agent.

\subsubsection{Statistical Analysis}

All results are expressed as means \pm SD. Statistical significance was judged employing Student's $t$ test for unpaired data and significance was defined as $p<0.05$. Analyses were conducted using SPSS for Windows version 16.0J for Windows (IBM, USA).

\section{Results}

\subsection{Effects of Riboflavin on Hcy and Folate Derivative Levels under Methionine Deficient Conditions}

As shown in Figure 2, the Hcy, 5-MTHF, and THF levels in supernatants did not change during the incubation period regardless of whether or not riboflavin was added. The 5,10-methenyl THF levels under incubation condition without riboflavin tended to be increased after 24 and 48 hours as compared to 0 hour, but were unchanged when riboflavin was added.

\subsection{Effects of Riboflavin Combined with 5-MTHF or THF on Hcy and Folate Derivative Levels under Methionine Deficient Conditions}

Under methionine-deficient but folate-supplemented conditions, low Hcy levels were maintained during the incubation period, regardless of whether or not riboflavin was added to the incubation medium (Figure 3). No differences were observed according to the type of folate added.

With the addition of 5-MTHF, the 5-MTHF level was markedly decreased, showing severe depletion, after 48 hours, while the THF level remained unchanged during the incubation period. These results were similar regardless of whether or not riboflavin was added to the incubation medium. The 5,10-methenyl THF level was increased at 24 hours followed by a decrease at 48 hours in the medium with only 5 -MTHF added, while higher levels were maintained by riboflavin addition $(\mathrm{p}<0.01)$ (Figure 3(A)).

With the addition of THF, 5-MTHF remained at an extremely low level. THF decreased markedly to one-third of the initial level at 24 hours, and this level was maintained at 48 hours regardless of whether or not riboflavin was added to the incubation medium. The 5,10-methenyl 

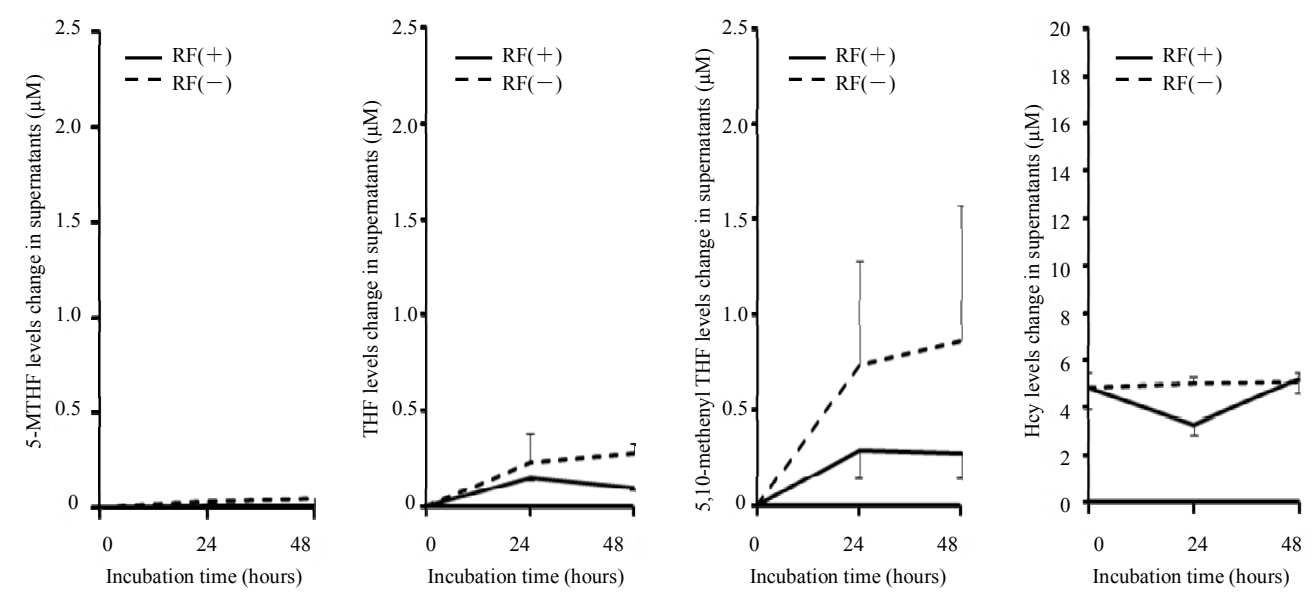

Figure 2. Effects of riboflavin on Hcy and folate derivative levels in rat hepatocytes. Accumulations of Hcy and folate derivatives in the medium were measured after hepatocytes had been cultured in basal medium (RF (-); dashed lines) or medium supplemented with $2.64 \mu \mathrm{M}$ RF $(\mathrm{RF}(+)$; solid lines) for 48 hours. Data are means \pm SD for 3 hepatocytes preparations.

(A) 5-MTHF added condition
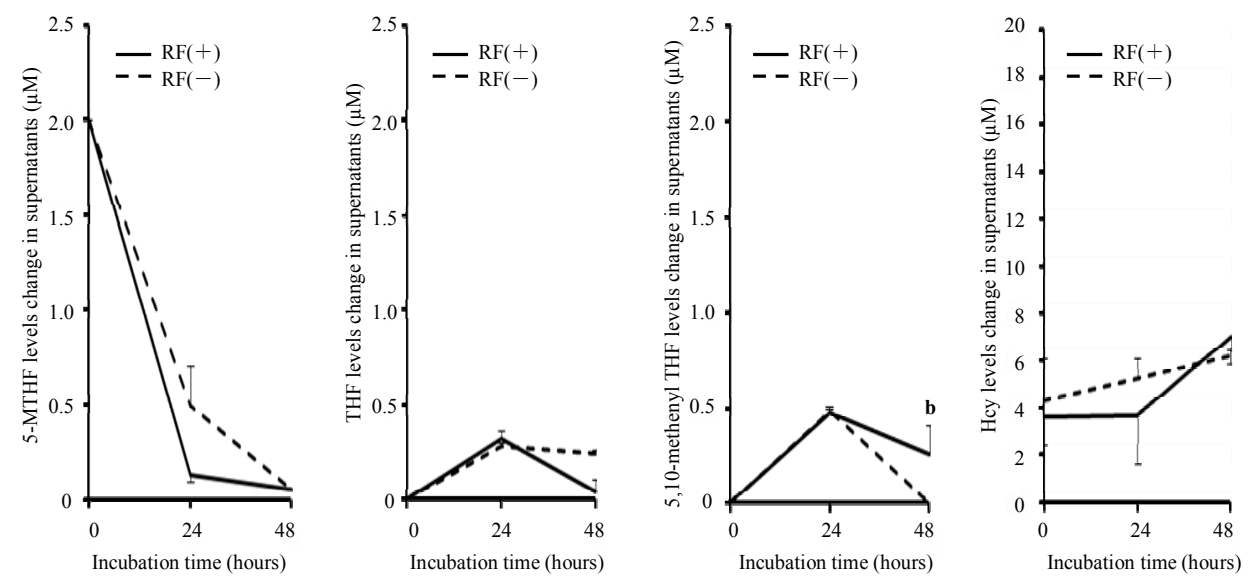

(B) THF added condition
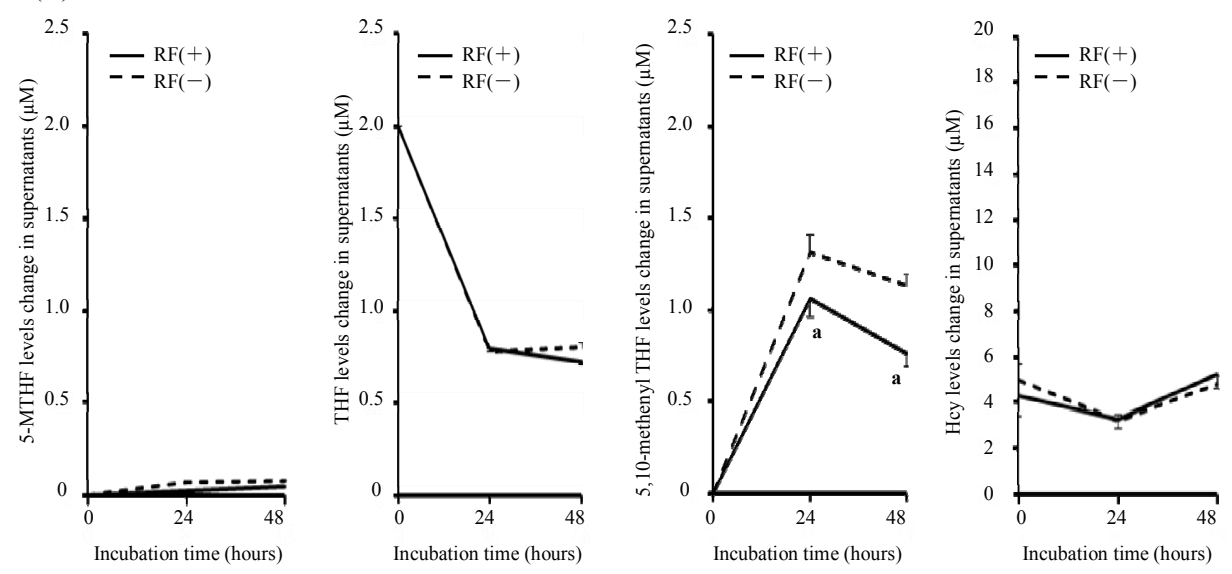

Figure 3. Effects of riboflavin combined with (A) 5-MTHF or (B) THF on Hcy and folate derivative levels. Accumulations of Hcy and folate derivatives in the medium were measured after hepatocytes had been cultured in basal medium supplemented with $2 \mu \mathrm{M}$ 5-MTHF or THF for 48 hours. In each experiment, two conditions, with $2.64 \mu \mathrm{M}$ riboflavin addition (RF(+); solid lines) and without riboflavin addition $\left(\mathrm{RF}(-)\right.$; dashed lines), were set. Data are means \pm SD for 3 hepatocytes preparations. ${ }^{\text {a,b }}$ : significantly different from the condition without riboflavin addition $\left({ }^{a}: p<0.05,{ }^{b}: p<0.01\right)$. 
THF level was increased at 24 hours and this level was maintained at 48 hours, with the levels being lower in the riboflavin-added medium than in that without riboflavin at both 24 and 48 hours $(\mathrm{p}<0.05)$ (Figure 3(B)).

\subsection{Effects of Riboflavin Combined with 5-MTHF or THF on Hcy and Folate Derivative Levels under Methionine-Loaded Conditions}

Under methionine-loaded conditions, the Hcy level was increased with a concomitant reduction in the 5-MTHF level throughout the incubation period in the riboflavin- deficient state. However, with the addition of riboflavin, the Hcy level did not increase and was actually lower than under the riboflavin deficient condition $(\mathrm{p}<0.05)$. The 5-MTHF level decreased to half of the initial level and was maintained above this level under riboflavin-added condition at 48 hours $(p<0.05)$. The THF level was increased after 24 hours and this level was maintained at 48 hours, while in the medium with riboflavin the THF level was lower than that without riboflavin at 24 hours $(\mathrm{p}<0.05)$. The 5,10-methenyl THF level did not change regardless of whether or not riboflavin was added to the incubation medium (Figure 4(A)).

(A) Methionine and 5-MTHF added condition
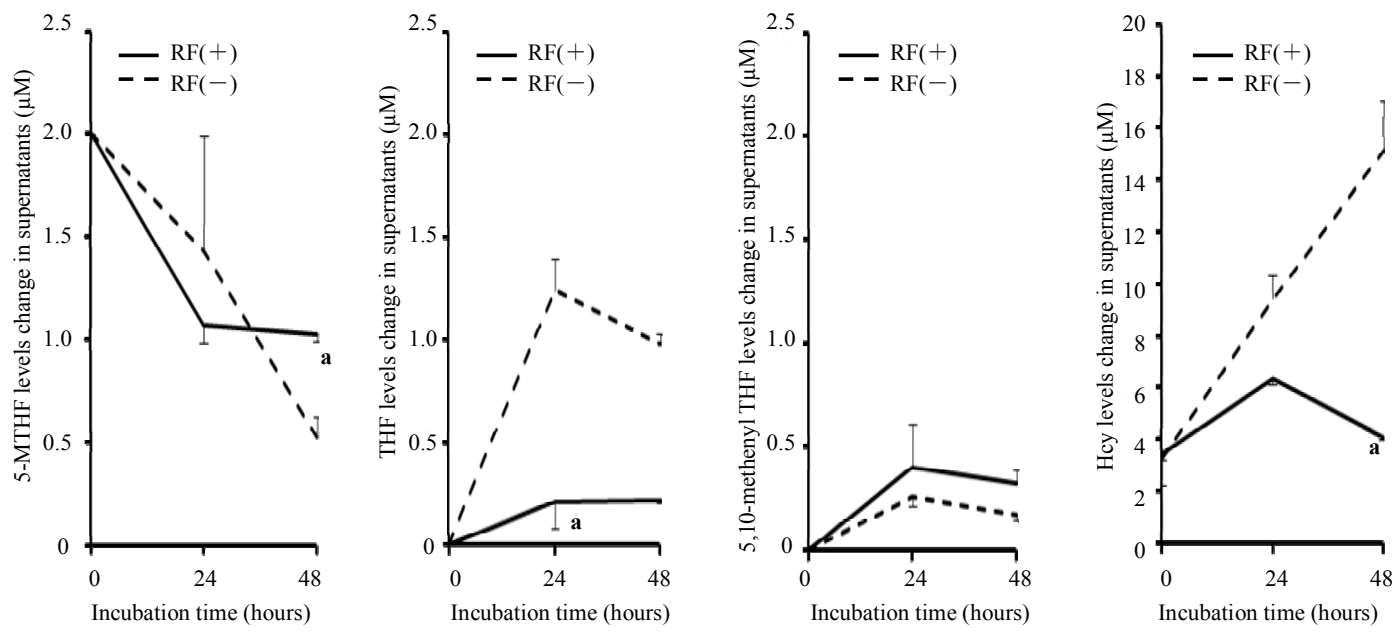

(B) Methionine and THF added condition
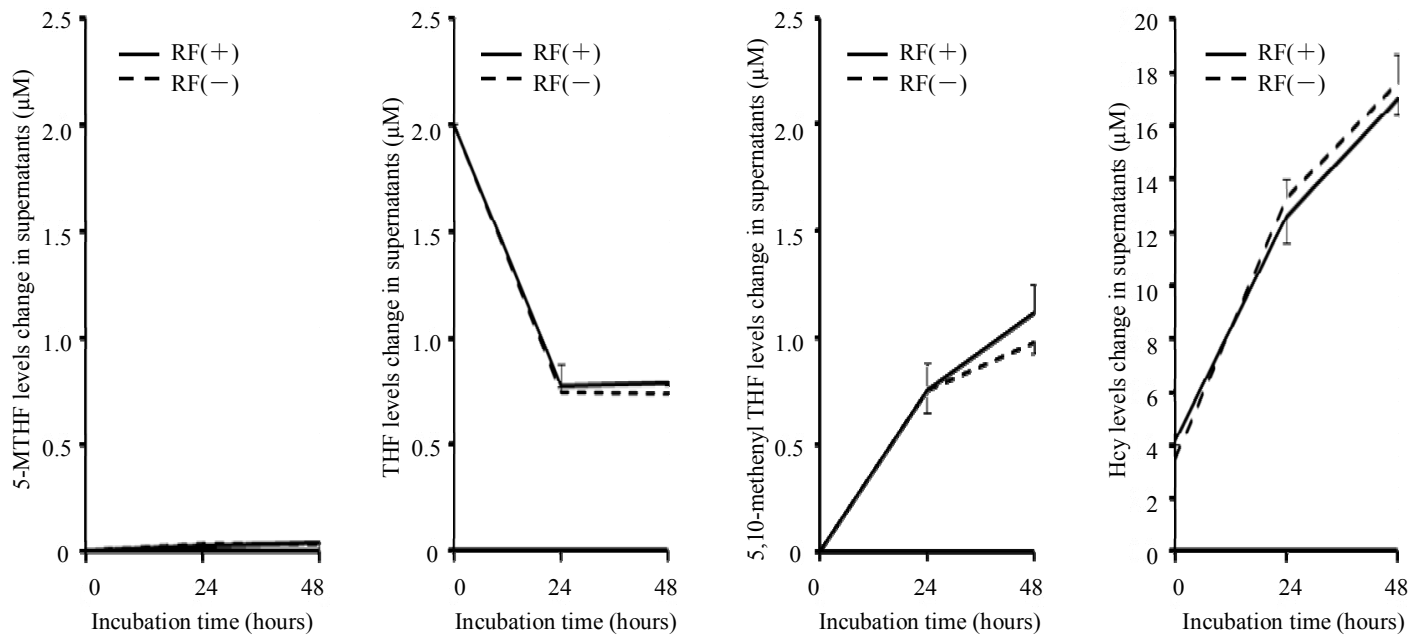

Figure 4. Effects of riboflavin combined with methionine and (A) 5-MTHF or (B) THF on Hcy and folate derivative levels. Accumulations of Hcy and folate derivatives in the medium were measured after hepatocytes had been cultured in basal medium supplemented with $5 \mathrm{mM}$ methionine and $2 \mu \mathrm{M}$ 5-MTHF or THF for 48 hours. In each experiment, two conditions, with $2.64 \mu \mathrm{M}$ riboflavin addition $(\mathrm{RF}(+)$; solid lines) and without riboflavin addition $(\mathrm{RF}(-)$; dashed lines), were set. Data are means \pm SD for 3 hepatocytes preparations. ${ }^{a}$ : significantly different from the condition without riboflavin addition $(p<0.05)$. 
With the addition of THF, the Hcy level was markedly increased after 24 hours and continued to rise during the entire incubation period. The 5-MTHF level showed no change, remaining at an extremely low level. The THF level showed a marked decrease to one-third of the initial level at 24 hours, and was maintained at this level through 48 hours. The 5,10-methenyl THF level continued to increase throughout the incubation period. Changes in Hcy, 5-MTHF, THF, and 5,10-methenyl THF levels did not differ between the incubation conditions with and without riboflavin (Figure 4(B)).

\section{Discussion}

MTHFR is a flavoprotein enzyme requiring a FAD, a derivative of riboflavin. Riboflavin would be expected to enhance the Hcy-lowering effect of folate. Thus, we examined the effects of riboflavin interactions with THF or 5-MTHF on changes in the levels of Hcy and folate derivatives in the present study.

To our knowledge, this is the first study to examine the effects of riboflavin alone and combined with 5-MTHF or THF on changes in the levels of Hcy and folate derivatives in rat primary hepatocytes. In prior studies using rat hepatocytes, significant amounts of methionine were added to EMEM so that Hcy could be recovered [14] and the dependence of Hcy export on methionine levels in rat hepatocytes in Krebs-Henseleit medium [11] has been reported.

The elevation of Hcy with methionine loading wasconfirmed in the present study (Figure 4). In the medium to which 5-MTHF had been added along with methionine loading markedly while the THF level increased. From these results, we can speculate that the added 5-MTHF was stored in THF form via the remethylation of Hcy. Taking into consideration that the Hcy level was not increased under methionine-deficient conditions, the added 5-MTHF might have been insufficient to suppress the Hcy increase due to methionine loading in the present study. However, even at the amount of 5-MTHF added, an enhanced Hcy-lowering effect, accompanied by inhibition of 5-MTHF reduction and increased THF, was observed with riboflavin addition. MTHFR activity might be inhibited by s-adenosyl methionine (SAM) which was increased by remethylation of Hcy with the addition of 5-MTHF, and the added riboflavin presumebly contributed to increased MTHFR activity. These changes likely resulted from accelerated conversion of THF to 5-MTHF via the preferentially increased MTHFR activity (Figure 4(A)).

An association between elevated Hcy and the risk of developing colorectal polyps [15], particularly in subjects with the TT genotype whose MTHFR activity is sensitive to riboflavin status [2], was previously reported. Furthermore, Powers et al. studied the effects of folic acid, with and without riboflavin supplementation, on Hcy and 5-MTHF status in healthy subjects and colorectal polyp patients, and demonstrated the plasma 5-MTHF increase to be enhanced by riboflavin supplementation only in subjects with polyps who had the MTHFR CT or the TT genotype [7]. These results suggested riboflavin supplementation to be beneficial under high Hcy conditions. Our observations of the effects of 5-MTHF and riboflavin combined on changes in Hcy and 5-MTHF levels under methionine-loaded condition support the results of Powers et al.

However, with addition of THF under conditions of methionine loading, there were no differences, between the presence and absence of riboflavin, in either folate derivative or Hcy levels. Hcy levels were markedly increased, to the same level as under 5-MTHF-added condition with methionine loading (Figure 4(B)). Its level was decreased but THF was not depleted, while 5,10methenyl THF continued to increase, and the 5-MTHF level was unchanged being nearly undetectable during the incubation period. Because the increased Hcy level did not exceed that under 5-MTHF-added condition, the reduced 5-MTHF metabolized from the added THF might have been used for remethylation of Hcy, and then regenerated to THF again. When folate is added as the THF form, the riboflavin requirement would be larger than when it is added in the 5-MTHF form. We should have measured riboflavin concentrations in the medium. Further study is needed to determine the effective amounts of THF and riboflavin for suppressing Hcy elevation under conditions of methionine loading.

THF is metabolized via two pathways. One involves direct synthesis of 5,10-methylene THF by serine hydroxymethyltransferase, and the other is conversion to 5,10-methylene THF via 10-formyl THF and 5,10-methenyl THF. The 10-formyl THF can be converted back into THF and used for the formylation of 5-amino-4-imidazole carboxamide ribonucleotide to 5-formamidoimidazole-4-carboxamide ribonucleotide (FAICAR). The FAICAR is then catalyzed to inosine monophosphate, which is involved in purine and pyrimidine metabolism [1]. The 5,10-methylene THF is a substrate not only for MTHFR but also for thymidylate synthesis with irreversible regeneration of THF, and riboflavin is required as a FAD precursor not only for MTHFR but also for the process of thymidylate synthesis [16].

Under conditions of THF addition, regardless of methionine loading, the THF level was decreased to some extent followed by a maintained the level while 5,10methenyl THF was increased (Figure 3(B), 4(B)). Thus, if there is a deficiency of 5-MTHF, the active form of 
folate, folate derivatives may be preferentially stored as reversible forms for purine metabolism regardless of Hcy accumulation. This hypothesis appears to be supported by the observation that the 5,10-methenyl THF level tended to increase under conditions with no additives (Figure 2).

However, with the addition of 5-MTHF, the 5,10methenyl THF level increase was suppressed to approximately half that under conditions with THF addition, while THF and 5,10-methenyl THF levels did not increase. It was not possible to ascertain, in the present study, whether regenerated THF from 5-MTHF is stored as other folate derivatives, such as 10-formyl THF, 5,10metyleneTHF and dihydrofolate, or used for purine synthesis.

In the complete absence of methionine, when supplemental folate was provided in the form of THF, storage of 5,10-methenyl THF was suggested to be suppressed by the addition of riboflavin, while it increased when 5-MTHF and riboflavin were both added to the medium. We can offer no explanation for the effects of riboflavin on the 5,10-methenyl THF level, which apparently differ depending on the form of added folate (Figure 2).

A limitation of this study is that we examined only a single amount of each additive, such that dose-dependent effects and those related to the relative amounts of additives could not be clarified. Furthermore, we examined only the levels of change in Hcy and folate derivat ives in the supernatant. The intracellular levels of Hcy and folate derivatives should also have been measured. It is not possible to assess changes in the levels of riboflavin, SAM, and folate derivatives under the conditions used in the present study. In addition, expressions of the genes for enzymes associated with folate, Hcy and nucleic acid metabolism should be studied. Thus, elucidation of the mechanisms underlying our results requires further study. Although much research remains to be conducted, the present results raise the following possibilities.

- Under conditions of methionine loading, supplementation with both 5-MTHF and riboflavin might enhance Hcy remethylation. However, this effect is less marked with THF supplementation.

- THF can be metabolized flexibly to 5,10-methylene THF and used in purine synthesis.

- The effects of riboflavin on 5,10-methenyl THF levels might differ between 5-MTHF and THF.

Appropriate folate intake is essential to health, but excess folic acid is reportedly associated with an increased risk of cancer [1]. Further examination is needed to reveal the effects of 5-MTHF and THF on changes in Hcy levels, including the interactions between riboflavin and nucleic acid metabolism.

\section{REFERENCES}

[1] A. D. Smith, Y. I. Kim and H. Refsum, "Is Folic Acid Good for Everyone?" The American Journal of Clinical Nutrition, Vol. 87, No. 3, 2008, pp. 517-533.

[2] H. McNulty, M. C. McKinley, B. Wilson, J. McPartlin, J. J. Strain, D. G. Weir and J. M. Scott, "Impaired Function of Thermolabile Methylenetetrahydrofolate Reductase Is Dependent on Riboflavin Status: Implications for Riboflavin Requirements," The American Journal of Clinical Nutrition, Vol. 76, No. 2, 2002, pp. 436-441.

[3] H. McNulty, L. R. C. Dewey, J. J. Strain, A. Dunne, M. Ward, A. M. Molloy, L. B. McAnena, J. P. Hughes, M. Hannon-Fletcher and J. M. Scott, "Riboflavin Lowers Homocysteine in Individuals Homozygous for the MTHFR 677CT Polymorphism," Circulation, Vol. 113, No. 1, 2006, pp. 74-80. doi:10.1161/CIRCULATIONAHA.105.580332

[4] S. J. Moat, P. A. Ashfield-Watt, H. J. Powers, R. G. Newcombe and I. F. McDowell, "Effect of Riboflavin status on the Homocysteine-Lowering Effect of Folate in Relation to the MTHFR (C677T) Genotype," Chinese Journal of Chemistry, Vol. 49, No. 2, 2003, pp. 295-302.

[5] R. Araki, C. Maruyama, S. Igarashi, M. Yoshida, T. Maruyama, T. Satoh, M. Yoshida and K. Umegaki, "Effects of Short-Term Folic Acid and/or Riboflavin Supplementation on Serum Folate and Plasma Total Homocysteine Concentrations in Young Japanese Male Subjects," European Journal of Clinical Nutrition, Vol. 60, No. 5, 2006, pp. 573-579. doi:10.1038/sj.ejen.1602351

[6] C. Z. Chuang, A. Boyles, B. Legardeur, J. Su, S. Japa and A. Lopez-S, "Effects of Riboflavin and Folic Acid Supplementation on Plasma Homocysteine Levels in Healthy Subjects," The American Journal of the Medical Sciences, Vol. 331, No. 2, 2006, pp. 65-71. doi:10.1097/00000441-200602000-00011

[7] H. J. Powers, M. H. Hill, M. Welfare, A. Spiers, W. Bal, J. Russell, Y. Duckworth, E. Gibney, E. A. Williams and J. C. Mathers, "Responses of Biomarkers of Folate and Riboflavin Status to Folate and Riboflavin Supplementation in Healthy and Colorectal Polyp Patients (the FAB2 Study)," Cancer Epidemiology, Biomarkers \& Prevention, Vol. 16, No. 4, 2007, pp. 2128-2135. doi:10.1158/1055-9965.EPI-07-0208

[8] M. Kimura, K. Umegaki, M. Higuchi, P. Thomas and M. Fenech, "Methylenetetrahydrofolate Reductase C677T Polymorphism, Folic Acid and Riboflavin Are Important Determinants of Genome Stability in Cultured Human Lymphocytes," Journal of Nutrition, Vol. 134, No. 1, 2004, pp. 48-56.

[9] L. L. Stern, B. Shane, P. J. Bagley, M. Nadeau, V. Shih and J. Selhub, "Combined Marginal Folate and Riboflavin Status Affect Homocysteine Methylation in Cultured Immortalized Lymphocytes from Persons Homozygous for the MTHFR C677T Mutation," Journal of Nutrition, Vol. 133, No. 9, 2003, pp. 2716-2720.

[10] J. D. Finkelstein and J. J. Martin, "Methionine Metabolism in Mammals. Adaptation to Methionine Excess," The Journal of Biological Chemistry, Vol. 261, No. 4, 1986, 
pp. 1582-1587.

[11] L. M. Stead, M. E. Brosnan and J. T. Brosnan, "Characterization of Homocysteine Metabolism in the Rat Liver," Biochemical Journal, Vol. 350, No. Pt 3, 2000, pp. 685692. doi:10.1042/0264-6021:3500685

[12] D. S. Rosenblatt, B. A. Cooper, S. Lue-Shing, P. W. K. Wong, S. Berlow, K. Narisawa and R. Baumgartner, "Folate Distribution in Cultured Human Cells. Studies on 5,10- $\mathrm{CH}_{2}-\mathrm{H}_{4}$ PteGlu Reductase Deficiency," Journal of Clinical Investigation, Vol. 63, No. 5, 1979, pp. 10191025. doi:10.1172/JCI109370

[13] D. W. Horne, "High-Performance Liquid Chromatographic Measurement of 5,10-Methylenetetrahydrofolate in Liver," Analytical Biochemistry, Vol. 297, No. 2, 2001, pp. 154-159. doi:10.1006/abio.2001.5334

[14] B. Christensen, H. Refsum, O. Vintermyr and P. M. Ueland, "Homocysteine Export from Cells Cultured in the
Presence of Physiological or Superfluous Levels of Methionine: Methionine Loading of Non-Transformed, Transformed, Proliferating, and Quiescent Cells in Culture," Journal of Cellular Physiology, Vol. 146, No. 1, 1991, pp. 52-62. doi: $10.1002 / j \mathrm{cp} .1041460108$

[15] F. P. Chen, C. C. Lin, T. H. Chen, M.C. Tsai and Y. C. Huang, "Higher Plasma Homocysteine Is Associated with Increased Risk of Developing Colorectal Polyps," Nutrition and Cancer, Vol. 65, No. 2, 2013, pp. 195-201. doi:10.1080/01635581.2013.756532

[16] X. Zhang, J. Zhang, X. Mao, Q. Zou, Y. Hu and D. C. Wang, "Crystallization and Preliminary Crystallographic Studies of a Flavin-Dependent Thymidylate Synthase from Helicobacter Pylori," Acta Crystallographica Section F: Structural Biology and Crystallization Communications, Vol. 66, No. Pt 5, 2010, pp. 513-515. doi:10.1107/S174430911000864X 